\title{
An extension of within-subject confidence intervals to models with crossed random effects
}

\author{
Stephen Politzer-Ahles ${ }^{\mathrm{a},} \otimes$ \\ ${ }^{a}$ 1Department of Chinese and Bilingual Studies, The Hong Kong Polytechnic University
}

\begin{abstract}
A common problem in displaying within-subject data is that of how to show confidence intervals that accurately reflect the pattern of significant differences between conditions. The Cousineau-Morey method (Cousineau, 2005; Morey, 2008) is a widely used solution to this issue; however, this method only applies to experimental designs with only one repeated-measures factor (e.g., subjects). Many experimental designs in fields such as psycholinguistics and social psychology use crossed random effect designs where, e.g., there are repeated measures both for subjects and stimuli. For such designs, extant methods for showing within-subject intervals would require first aggregating over stimuli, and thus such intervals might be a less accurate reflection of the statistical significance patterns if the data are actually analyzed using a method that takes both random effects into account (e.g., linear mixed-effects models). The present paper proposes an extension of the method described above to address this problem; the proposal is to scale the data using a mixed-effects model, rather than using the means from each subject, and then calculate confidence intervals from the data scaled thusly. Analysis of a sample of crossed random effect datasets reveals that intervals calculated using this method give a slightly more accurate reflection of the pattern of statistical significance in the between-condition differences.
\end{abstract}

Keywords a confidence intervals, repeated measures, linear mixed effects, within-subjects confidence intervals, crossed random effects.

stephen.politzerahles@polyu.edu.hk

SPA: 0000-0002-5474-7930

Acting Editor $\square$ Denis Cousineau (Université d’Ottawa)

\subsection{2/tqmp.13.1.p075}

\section{Introduction}

Displaying informative summaries of data is a common challenge in experimental psychology. One useful tool for displaying and interpreting data is the confidence interval (see, e.g., Cumming, 2014), which allows readers to see not just a single parameter of a dataset (e.g., the mean), but also an estimate of what that parameter differs from statistically. Compared to a single mean, a confidence interval is more useful for showing what null hypotheses are consistent with that parameter and what null hypotheses can be ruled out. ${ }^{1}$

There is a challenge, however, when it comes to using confidence intervals to represent a pattern of signif- icant differences between conditions. For a design with two conditions to be compared, the only way to accurately show the significance of the between-condition difference is to plot the confidence interval of the difference (which Rouder and Morey, 2005, refer to as a "relational CI"), not the confidence intervals of each condition's mean (which Rouder and Morey refer to as an "arelational CI"; see Blouin \& Rioppele, 2005; Cumming \& Finch, 2005; Franz $\&$ Loftus, 2012). For example, for the data shown in Figure 1 , confidence intervals of each condition's mean (panel B) are uninformative with respect to whether the differences between conditions are significant, but confidence intervals of the pairwise differences (panel $\mathrm{C}$ ) reveal that each pair of conditions is significantly different, as the intervals

\footnotetext{
${ }^{1}$ Confidence intervals are often said to be useful for indicating the precision of a parameter estimate or indicating the plausible values of a parameter, but these inferences are not strictly justified (Morey, Hoekstra, Rouder, \& Wagenmakers, 2016); confidence intervals are related to null hypothesis statistical testing and do not assign likelihoods.
} 
Figure 1 I Example of within-subjects intervals. (A) Sample repeated-measures data from Loftus and Masson (1994); each line represents one subject's data in three conditions. (B) Standard confidence intervals calculated across participants for each condition, which do not accurately reflect that the between-condition differences are statistically significant in a repeated-measures analysis. (C) $95 \%$ confidence intervals of the difference between each pair of conditions. (D) The data scaled by subtracting the subject's mean from each data point and adding back the grand mean, which removes between-subject baseline variability and preserves just the pattern of within-subject differences. (E) 95\% within-subject intervals calculated using the method described by Cousineau (2005) and updated by Morey (2008), which are narrower and more accurately reflect the significant between-condition differences.
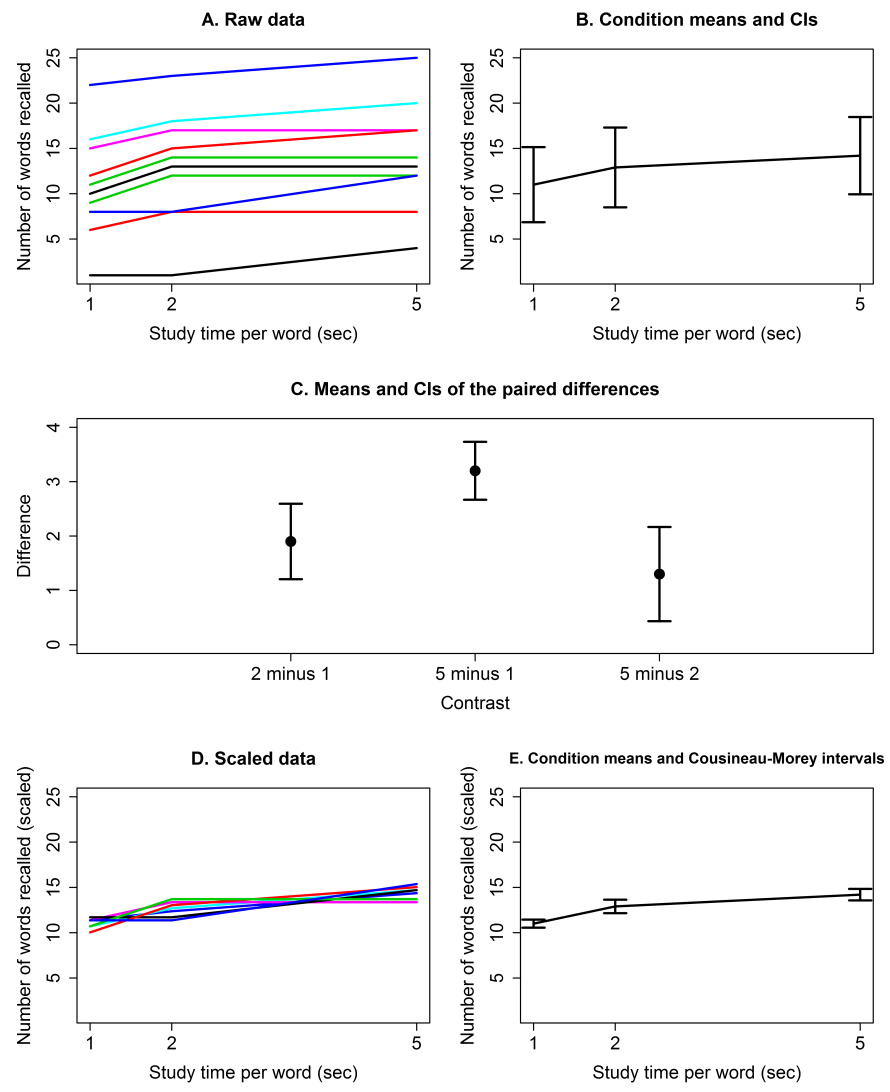

do not cross zero.

However, plotting each pairwise difference between conditions is not always a feasible way to display data. When plotting the results of an experiment with a large number of conditions, showing all possible pairwise comparisons may be prohibitive in terms of space or complexity. A design with $K$ levels has $(K(K-1)) / 2$ pairwise differences, so an experiment with, e.g., 8 conditions has 28 pairwise differences (plus main effects and interactions, in the case of a factorial design) that could be shown. Even with a small number of conditions, standard practice in many fields is still to show each condition mean, rather than each difference.

One such field is psycholinguistics. I reviewed pa-

pers published within the last year in two highly-regarded psycholinguistics journals, Journal of Memory and Language $(N=39)$ and Language, Cognition and Neuroscience $(N=46)$ and found that, of 121 relevant figures and tables that showed any kind of error bar, 92 (76\%) showed only individual conditions, not between-condition differences (see Supplementary File 1; 163 figures and tables are listed there, but 42 of these did not show or describe any sort of error bar and thus are not discussed here). Of the 29 figures and tables that did show betweencondition differences, 21 of these (72\%) were regression tables with coefficients and standard errors. Only 4 papers in the sample directly showed between-condition differences in figures. Nevertheless, practitioners often attempt 
to use the pattern of confidence interval overlap to make inferences about which pairs of conditions have significantly different means. For example, one paper made explicit reference to the pattern of confidence interval overlap in the interpretation of between-condition differences (Gould, McKibben, Ekstrand, Lorentz, \& Borowsky, 2016, p. 243), "The means of two conditions are considered statistically different if the mean of one condition is not captured by the $95 \%$ CI of the other condition"-which is not strictly correct for the type of confidence interval used in this example), and indeed Belia, Fidler, Williams, and Cumming (2005) have provided empirical evidence that researchers rely (often erroneously) on comparing whether arelational confidence intervals of condition means overlap in order to make conclusions about significant differences.

What is often desirable is a way to show each condition's mean (or some other relevant sample statistic) along with an interval that allows rough inferences about which conditions it differs from. A reader can then compare separate confidence intervals of two different conditions to see how much they overlap; while this is only a rough heuristic for judging whether the two conditions differ (Cumming \& Finch, 2005), it can be a more efficient means of displaying data compared to showing all pairwise differences. Rouder and Morey (2005) likewise recommend the display of individual means along with [arelational] confidence intervals, rather than or in addition to display of the differences, for parsimonious data visualization, while reminding that these need to be supplemented with direct statistical comparisons.

\section{The reason for within-subject intervals}

On top of this issue, an additional challenge is raised when it comes to displaying confidence intervals for withinsubjects designs (for review see Baguley, 2012; Blouin \& Rioppele, 2005; Cousineau, 2005; Franz \& Loftus, 2012; Loftus \& Masson, 1994; Morey, 2008). As Loftus and Masson (1994) demonstrate, when a dataset includes conditions with repeated measures (e.g., when a single participant contributes a data point to each condition in an experiment), then standard confidence intervals around each mean are even less informative for illustrating the pattern of significant differences between conditions. Loftus and Masson describe a memory experiment in which each participant attempts to recall words in different conditions; while the within-subject differences between conditions are very systematic, there is large between-subject variance in the average number of words recalled across conditions, and thus the standard confidence intervals for the conditions are very wide, overlapping substantially and giving the impression that the between-condition differences are not significant (Figure 1B). While this problem could be resolved by showing the confidence intervals of the between-condition differences instead of the confidence intervals of each condition's mean (as done in Figure 1C), this is rarely done, and in some cases it is not feasible, as described above.

As another way to address this problem of withinsubjects data, several methods have been proposed for calculating within-subjects intervals (see Baguley, 2012, for review). ${ }^{2}$ What these methods have in common is that they adjust the size of a confidence interval such that they only reflect variance in within-subject effects; whether or not two confidence intervals sufficiently overlap then roughly corresponds to whether or not the difference between the associated conditions is nonsignificant. While the intervals produced by these methods are not true confidence intervals, and are subject to Cumming and Finch's (2005) caveats about trying to compare two confidence intervals, they can be useful for making a quick visual summary of the dataset that allows very rough inferences about which pairwise comparisons will probably be significant. One type of within-subject interval (Cousineau-Morey intervals) is shown in Figure 1E.

\section{The problem of crossed random effects}

The proposed methods, however, have thus far only been implemented for designs with a single random effect, typically Subjects (hence the name "within-subject intervals"). Many research paradigms, however, have multiple random effects. In psycholinguistics, for example, experiments that cross Subjects and Items (or "Stimuli") are common (Baayen, Davidson, \& Bates, 2008; Chang \& Lane, 2016; Judd, Westfall, \& Kenny, 2012). For instance, a semantic priming experiment might examine whether people respond faster to words presented on a screen (e.g., $D O C T O R$ ) if the word is preceded by a related word (e.g., nurse) rather than an unrelated word (e.g., table). Usually such an experiment not only contains multiple subjects, but also multiple words. Just as each subject will contribute data points in each condition (i.e., a given subject will complete both Related and Unrelated trials), so will each word (e.g., DOCTOR may appear in both Related trials and Unrelated trials). In such an experiment, both Subjects and Items (words) are random effects with repeated measures. Extant methods for calculating within-subject intervals would require first aggregating over items to get condition means for each subject, or aggregating over subjects

\footnotetext{
${ }^{2}$ Throughout this paper I refer to these as " intervals" rather than "confidence intervals" because, as noted originally by Loftus and Masson (1994), these intervals are not true confidence intervals (i.e., it is not the case that if you repeat the experiment 100 times then 95 of the $95 \%$ confidence intervals would contain the population parameter).
} 
Figure 2 - Simulated data from a semantic priming experiment with a design that is both within subjects and within items. (A) Pairwise differences (Unrelated minus Related) for each subject and for each item (one dot represents one subject or one item); error bars show the standard 99\% confidence interval of the mean of the differences. (B) Condition means shown together with error bars depicting several types of interval at the $99 \%$ confidence level.

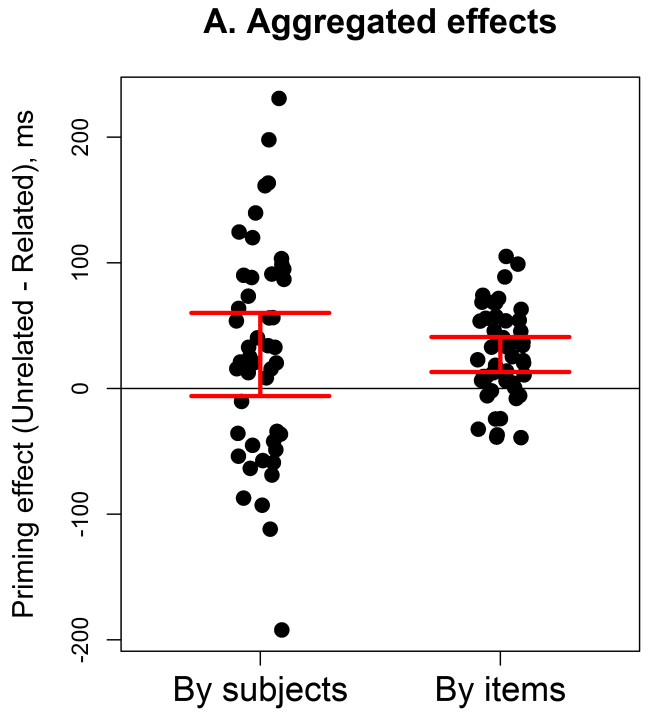

to get condition means for each item, and then calculating within-subject intervals (or within-item intervals) across those aggregated means. This, however, would not be an accurate reflection of the actual statistical analysis of these data: for the past decade, the field of psycholinguistics has been moving away from using separate by-subject and byitem analyses, and towards using linear mixed effect models that take into account both subject and item variance simultaneously (Baayen et al., 2008; Chang \& Lane, 2016). Thus, if a researcher intends to display within-subject intervals to summarize experimental data from a repeatedmeasures paradigm, it would be ideal to use intervals that reflect the corresponding statistical analysis as accurately as possible (while keeping in mind the limitation that such intervals are still only a rough heuristic and are not true confidence intervals).

This problem is illustrated in Figure 2, which shows simulated data from a semantic priming paradigm like that described above (the data are available in Supplementary File 2). As shown in panel A, the priming effect (the difference between unrelated and related prime conditions) in this dataset is substantially more variable across subjects than it is across items. Therefore, while the effect is in fact close to significant at the $\alpha=.01$ level $(b=27.04$,

\section{B. Condition means with intervals}

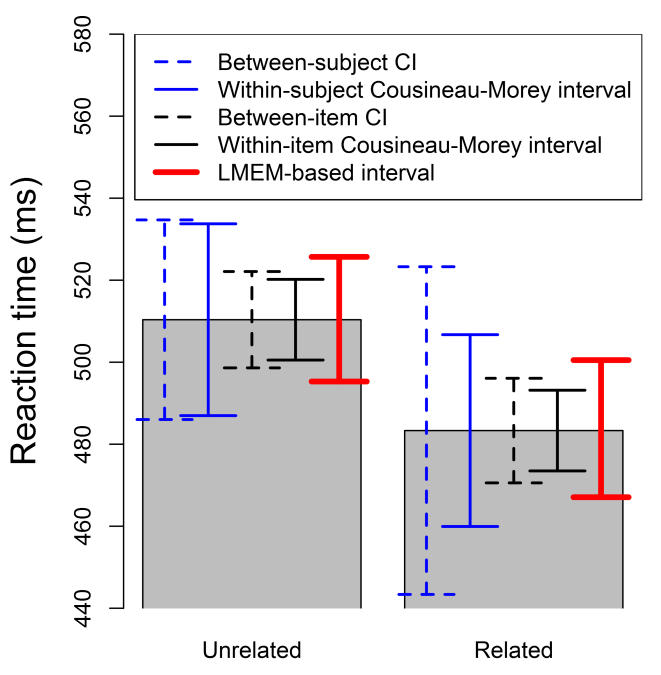

$S E=12.48, t=2.17$, when using a maximal linear mixed-effects model - Barr, Levy, Scheepers, and Tily, 2013), the $99 \%$ confidence interval of the by-subject effects nevertheless crosses zero. This problem could be avoided by showing the confidence interval of the coefficient (i.e., the Unrelated-Related difference) from the mixed effects model; however, as discussed above, researchers often desire to show each condition mean rather than the mean and confidence interval of the pairwise difference. The problem of crossed random effects also affects the plot of condition means: as shown in panel B, the within-subject (solid blue) and within-item (solid black) intervals look very different, with the non-overlapping within-item intervals suggesting a significant difference whereas the substantially overlapping within-subject intervals suggest a non-significant difference. If a researcher created a plot showing only within-subject intervals, the significance pattern suggested by the figure would be inconsistent with the results of inferential statistics.

Previous methods for calculating within-subject intervals (e.g., the Cousineau-Morey method and the Loftus \& Masson method) were never intended to be used in designs with crossed random effects; therefore, their inapplicability to the sorts of data described above is not a bug, but a 
feature. Nonetheless, such designs are common in cognitive psychology. Therefore, new methods that allow practitioners to visualize patterns of significant differences, in a similar way as allowed by within-subjects intervals, would be of value.

\section{A solution: linear mixed effect model residuals}

One solution to the problem of crossed random effects is to scale the dependent variable using a linear mixed effects model. To motivate this solution, it is informative to compare it to the scaling method used to calculate CousineauMorey intervals (Cousineau, 2005; Morey, 2008), illustrated in Figure 1D.

Deriving Cousineau-Morey intervals involves the following steps:

1. Scale the data to remove the between-subject baseline differences. This is done by calculating the mean of the dependent variable (e.g., number of words recalled in Figure 1, or reaction time in the semantic priming example) for each participant. The relevant participant's mean is then subtracted from each data point, preserving the within-participant pattern of effects but removing between-participant baseline differences.

2. Add the original (prior to scaling) grand mean back to each data point to ensure that the overall dataset has the same mean as the original data, simply with between-subject variance removed.

3. Calculate within-subject intervals from the scaled data using the typical formula for a confidence interval (times a bias-removing scaling factor described by Morey, 2008).

It should be clear that the first step of this process, subtracting the relevant participant's mean, is equivalent to residualization. In regression, the difference between an observed value and the predicted value is a residual. When no other predictors are present, the predicted value for a given participant is that participant's mean. Thus, subtracting the participant's mean from each of that participant's data points is exactly the same as regressing the dependent variable on Subject (treated as a fixed factor) and extracting the residuals. The code given in Listing 1 demonstrates that this is true, using the sample data from Loftus and Masson (1994); see Figure 1 and discussion above,. It shows that the scaled values obtained by subtracting each participant's mean are exactly the same as the regression residuals ( $r=1$ ).

Just like ordinary least squares regression, mixed effects regression can also return residuals. (These residuals, though, from a model in which subjects are treated as a random effect, will not be exactly the same as residuals from a model treating subjects as a fixed effect, which is essentially what is done in the Cousineau-Morey and Lof- tus \& Masson methods. This is because random effects in a mixed effect model undergo shrinkage: rather than reflecting the actual mean for that subject or item, the best linear unbiased predictors for the subject and item intercepts [i.e., the estimates of how much a given subject's or item's mean deviates from the grand mean] are constrained to be somewhat closer to overall intercept of the model; see e.g. Blouin and Rioppele, 2005.)

Importantly, mixed effects regression residuals can be calculated from a model that includes both subjects and items as random effects. Thus, there is no need to aggregate over subjects or items. Rather, the data can be scaled in a way similar to the way data are scaled in the Cousineau-Morey method (step 1 above): fit a model with only a fixed intercept and with random intercepts for subjects and items, extract the residuals, and add the grand mean of the original data. In fact, as Blouin and Rioppele (2005) note, methods that calculate within-subject intervals based on treating subjects as fixed effects (including the Cousineau-Morey and Loftus \& Masson methods) are in fact just a special case of methods that use a mixed effect model; the present proposal builds on their observation by extending this concept to mixed effect models with more than one random effect.

\section{How to calculate intervals from scaled data}

The next problem is how to use these scaled data to compute an interval. The Cousineau-Morey method simply uses a variation of the standard formula for a confidence interval:

$$
\bar{x} \pm\left(s d(x) / \sqrt{N} \times \operatorname{tinv}_{1-\frac{\alpha}{2}}, \quad N-1 \times M\right)
$$

where $x$ is the scaled data, $N$ is the number of participants, tinv is the quantile function for the $t$ distribution (returning the critical $t$ value for a given significance level and degrees of freedom), and $M$ the correction factor from Morey (2008). This method is not applicable for designs from a mixed effect model, however, as the standard formula relies on calculating the critical $t$ statistic for a given number of degrees of freedom $(N-1)$, whereas the degrees of freedom for a mixed effect model are not known (Baayen et al., 2008). There are methods available to estimate the degrees of freedom, but the best option for the present application is to calculate bootstrap-based confidence intervals of the scaled data for each condition (Davison \& Hinkley, 1997) rather than using a confidence interval formula. Bootstrapping for mixed effect models is straightforwardly implemented in the bootMer package of the $\mathrm{R}$ statistical environment. The LMEMinterval () function shown in Appendix B on page 87 is a convenience function which scales the dependent variable of a dataset using mixedeffect model residuals, and then bootstraps the dependent

The Quantitative Methods for Psychology 
variable to construct an interval; the mixed-effect-modelbased intervals shown in Figure 2B were derived using this function.

\section{Implementation and code}

As mentioned above, Appendix $\mathrm{B}$ at the end of this article contains $\mathrm{R}$ code for a convenience function, LMEMinterval (), that calculates bootstrap mixed effect model intervals using the procedure described above. This code assumes that the random effects are crossed, and there is no guarantee that it will work for between-subject or between-item designs. It also requires that the lme4 package is installed.

To use the function, you must pass it a data frame and a formula using the same formula syntax used in the 1 me 4 package. See Baayen et al. (2008), Chang and Lane (2016), inter alia, for introductions to the lme4 syntax.

By way of example, the following command may be used to read the data from the semantic priming experiment discussed above (Supplementary File 2) into R (after having saved the file to a local drive, and after having run the code in Appendix B to have the LMEMinterval () function in R's memory for the current session) and then calculate the LMEM-based confidence intervals (this code will take several minutes to run, and may issue several failure-to-converge warning messages which may be ignored):

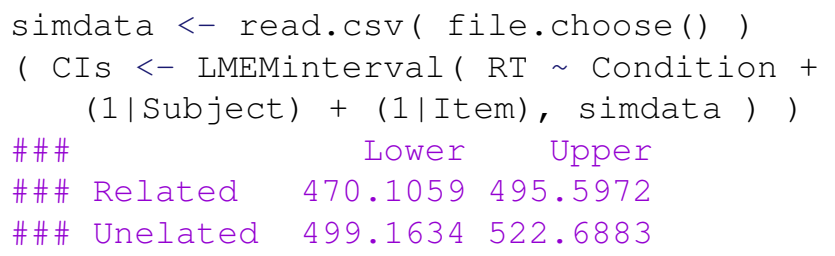

(Because of the randomness involved in bootstrapping, the results will be slightly different each time the function is called.) The confidence level may be changed using the conf parameter, e.g., conf $=.99$ will calculate a $99 \%$ rather than a $95 \%$ interval. The number of bootstrap replicates used for calculating the bootstrap interval may be changed using the nsim parameter. The number of bootstrap replicates is the main determinant of how long the function call will take. The default is to use 2000 replicates for a percentile bootstrap. To make the function run faster, you can request a normal bootstrap instead of a percentile bootstrap, which by default uses only 200 replicates:

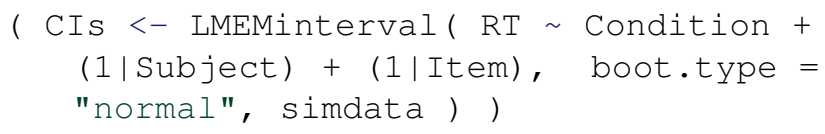

The function can straightforwardly be used for other datasets as well. For instance, the following example demonstrates the use of the function with the lexdec dataset that is included in the languager package.

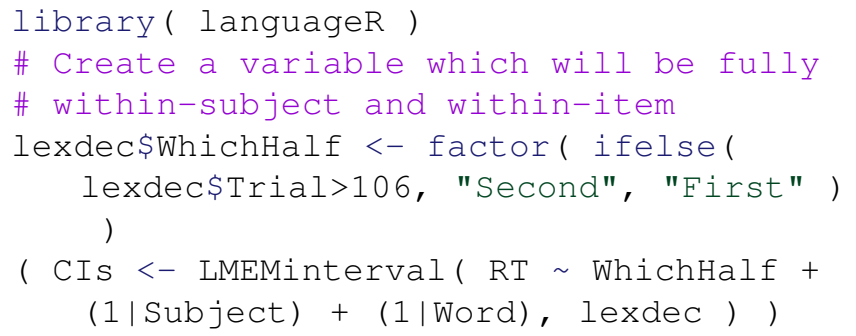

\section{Testing the method}

It is worthwhile to test whether mixed effect intervals are in fact more accurate summaries of the data pattern than other types of within-subject interval. In what follows, I use several real datasets (a convenience sample including data from published psycholinguistics papers, datasets available online, and data from unpublished experiments) to compare mixed effect intervals, within-subject and within-item Cousineau-Morey intervals in terms of how closely they match the p-values obtained from a direct comparison between the conditions of interest.

\section{Evaluation criteria}

A metric is needed to determine how accurate an interval is. One appropriate metric is whether the overlap between two conditions' intervals accurately corresponds to a $p$ value. As Cumming and Finch (2005) note, as long as several assumptions about the distribution of the data hold, two independent groups are usually different at the 5\% significance level if their respective $95 \%$ confidence intervals overlap by just about $58 \%$ (specifically, $|\sqrt{2}-2|$; see footnote 3 ) of the average margin of error (the margin of error is half of the confidence interval-for a symmetrical confidence interval, the margin of error is the distance from the parameter estimate to the lower or upper bound of the interval-and the average margin of error is the average of

\footnotetext{
${ }^{3}$ This $58 \%$ overlap rule is related to the standard error of the difference between means. While the standard error for a single sample mean with $\mathrm{N}$ observations is $S D / \sqrt{N}$, the standard error of a difference between sample means uses a different formula (see, e.g., Pfister \& Jancyzk, 2013, equation 4). In a case where the two sample means have the same $\mathrm{N}$ and same variance, the formula for the standard error of the difference can be algebraically reduced to $S D / \sqrt{N} \times \sqrt{2}$. If the margin of error for the confidence interval of a sample mean is multiplied by $\sqrt{2}$, then the difference between means is significant if the confidence interval does not include the other mean, rather being significant if the two confidence intervals overlap by less than $58 \%$ of the average margin of error as above. To illustrate, consider a hypothetical case of two means of unpaired samples where each sample has the same $\mathrm{N}$ and same variance. The margin of error (MoE) can be calculated based on the standard error of each mean (and in this example the average MoE will equal the MoE for either mean, since their variances and Ns are the same) or on the standard error of the difference. Assuming $\bar{x}_{1}$ is the lower of the two sample means and $\bar{x}_{2}$ is the higher, then the critical MoE of the difference (i.e., the margin of error such that the difference between means will
} 
two condition's respective margins of error). ${ }^{3}$ This applies to any significance level; for instance, if the $p$-value for the difference between two groups is .2, then the two groups' $80 \%$ confidence intervals should overlap by just about $58 \%$ of the average margin of error, if the right assumptions are met. The same rough heuristic applies to two paired groups and their Cousineau-Morey intervals (a demonstration of this, using simulations, is given in Appendix A). Therefore, we can test the accuracy of mixed effect intervals by calculating, for any given pairwise comparison in a crossed random effect dataset, what the $p$-value of that comparison is, and what is the largest interval that overlaps by just about $58 \%$ of the margin of error. For example, if the $p$-value for the difference between two conditions in a mixed-effect model is .003 , and the $99.7 \%$ mixed effect based intervals of the two condition means overlap by $58 \%$ of the average margin of error, then the intervals are consistent with the $p$-value; on the other hand, if the $99.7 \%$ intervals overlap by much less or much more than that, then the intervals are inconsistent with the actual $p$-value.

\section{Sample}

A convenience sample of 11 psycholinguistic studies with subject and item random effects, a continuous dependent variable, and at least one nominal independent variable was selected. This independent variable was repeated within subjects and items. Some of these studies also involved between-subjects or between-items variables; in these cases, these were treated as separate experiments and LMEM-based intervals for each group were calculated separately (for example, if a study included two conditions that were measured within both subjects and items, but also had a Group variable that was between-subjects, then this was treated as two separate datasets, one for each group). This was done to avoid the complications in creating appropriate intervals for a design involving both within-subjects and between-subjects comparisons. Across these studies, 162 pairwise comparisons were available to compare the performance of different types of intervals. The samples are summarized in Table 1; links to full citations are available in Supplementary File 4.

\section{Calculations}

Supplementary File 3 includes $\mathrm{R}$ code that compares the actual $p$-value to the $p$-value suggested by the interval, for all possible pairwise contrasts within any given dataset. (This code is only included for demonstration purposes; for calculating mixed-effect intervals under normal circumstances, the reader is advised to use the LMEMinterval ( ) function included in Appendix B.) Because the exact $\mathrm{p}$-values for mixed effect model contrasts are not known, this script calculates several $p$-value approximations:

1. percentile bootstrap p-values (2 times the percentage of bootstrap beta values for that contrast which fall on the wrong side of zero; for example, if the estimate for a difference is 3, and forty of 2000 bootstrap replicates give an estimate below zero, then the bootstrap $p$-value is $2 \times 40 / 2000=.04$ );

2. p-values based on the Satterthwaite approximation for mixed effect model degrees of freedom (Schaalje, McBride, \& Fellingham, 2002);

3. p-values based on treating the $t$ statistic as a $z$ statistic (because the $t$ distribution approaches the normal distribution when the number of degrees of freedom is high).

It then reports the corresponding interval-based $\mathrm{p}$ values (i.e., the confidence levels of the widest intervals that overlap by just $58 \%$ of the margin of error) for three types of interval:

1. within-subject Cousineau-Morey intervals;

2. within-item Cousineau-Morey intervals;

3. mixed effect intervals.

The crucial comparison is that between the "real" $p$ value (the bootstrap-based $p$-value of the actual difference between conditions) and the $p$-value suggested by how much the mixed-effect intervals overlap (e.g., if the $85 \%$ intervals for two conditions overlap by $58 \%$, then this suggests a $p$-value of about $1-.85=.15$ for the direct comparison between these two conditions). If mixed effect intervals are indeed more accurate, they should show a closer correspondence to the $\mathrm{p}$-values.

be just barely significant at the chosen alpha level) is the one that is equal to or just below the size of the difference between means, or in other words, the difference between means is equal to the MoE. Keeping in mind that the MoE of the difference is, in this example, equal to the MoE of either mean times $\sqrt{2}$, this means that the critical margin of error is as follows: $\left(\bar{x}_{2}-\bar{x}_{1}\right)=M o E \times \sqrt{2}$. This can be solved for the MoE: $\left(\bar{x}_{2}-\bar{x}_{1}\right) / \sqrt{2}=M o E$. On the other hand, when using the MoE of each mean, the critical MoE is the one such that the confidence intervals overlap by .58 times the average MoE: $\left(\bar{x}_{1}+M o E\right)-\left(\bar{x}_{2}-M o E\right)=.58 \times M o E$. This can be solved for the MoE: $\left(\bar{x}_{2}-\bar{x}_{1}\right) / 1.42=M o E$. As $\sqrt{2} \approx 1.42$, this demonstrates the motivation behind using $58 \%$ overlap as an approximate value to judge statistical significance-it corresponds to the $\sqrt{2}$ used for calculating a confidence interval of a difference. Indeed, instead of estimating whether two confidence intervals overlap by 58\% the average MoE, another approach to using confidence intervals to roughly visualize statistically significant differences is to multiply the MoEs by $\sqrt{2}$, creating difference intervals that imply significance (except for the caveats mentioned above, if the sample variances or sample sizes differ) when the interval for one mean does not include the estimate of the other mean. 
Table 1 - Summary of the studies used to in the present test. Links to datasets are available in Supplementary File 4.

\begin{tabular}{|c|c|c|c|c|c|}
\hline \multirow[b]{2}{*}{ Source } & \multirow[b]{2}{*}{ Publication status } & \multicolumn{4}{|c|}{ Number of } \\
\hline & & subjects & items & conditions & $\begin{array}{l}\text { pairwise } \\
\text { comparisons }\end{array}$ \\
\hline Politzer-Ahles and Fiorentino (2013) & published & 28 & 48 & 4 & 6 \\
\hline Politzer-Ahles and Husband (2016) & unpublished & 48 & 48 & 4 & 6 \\
\hline Husband and Politzer-Ahles (2016) & unpublished & 48 & 48 & 6 & 15 \\
\hline Baayen (2008) & online dataset (CRAN) & 21 & 79 & 2 & 1 \\
\hline Politzer-Ahles (ms) & unpublished & 40 & 24 & 4 & 6 \\
\hline Politzer-Ahles and & published & 25 & 12 & 4 & 6 \\
\hline \multirow[t]{3}{*}{ Zhang (in press) } & & 25 & 12 & 4 & 6 \\
\hline & & 22 & 12 & 4 & 6 \\
\hline & & 22 & 12 & 4 & 6 \\
\hline Politzer-Ahles and Connell (dataset) & unpublished & 25 & 12 & 4 & 6 \\
\hline Fruchter et al. (2015) & published & 103 & 158 & 2 & 1 \\
\hline Matushanskaya & online dataset (OSF) & 24 & 40 & 6 & 15 \\
\hline et al. (dataset) & & 24 & 40 & 6 & 15 \\
\hline Gibson and Wu (2012) & Published & 37 & 15 & 2 & 1 \\
\hline Mädebach et al. (dataset) & online dataset (OSF) & 24 & 32 & 12 & 66 \\
\hline
\end{tabular}

Note. Some studies were broken down into multiple smaller studies because they included between-subjects and/or between-items manipulations; see text.

\section{Results}

Supplementary File 4 shows these various p-values from all within-subject-and-item contrasts in the sample. On average, the p-values implied by the LMEM-based intervals deviated from the actual bootstrap p-values by .017 $(\mathrm{SD}=.026)$; these are shown in Column M of Supplementary File 4 . On the other hand, p-values implied by withinsubject Cousineau-Morey intervals differed from actual pvalues by an average of .035 ( $\mathrm{SD}=.059$; Column $\mathrm{N}$ ), and $\mathrm{p}$ values implied by within-item Cousineau-Morey intervals by .050 ( $S D=.080$; Column O). For instance, to take one representative example: for the fourth comparison in PolitzerAhles and Fiorentino, 2013 (the fifth row of Supplementary File 4), the bootstrap $p$-value for the difference between these conditions in a mixed-effect model directly comparing them is .352. The $p$-value suggested by LMEMbased intervals is .36125 (i.e., it is the $63.875 \%$ intervals that overlap by just about $58 \%$ of the average margin of error), which deviates from the real $p$-value by .00925. On the other hand, the $p$-value suggested by within-subjects
Cousineau-Morey intervals is .3065 (deviating from the real $p$-value by .0455), and that suggested by within-items Cousineau-Morey intervals is .301 (deviating by .051). Figure 3 shows the distribution of these differences for the entire sample of 162 pairwise comparisons; it can be seen that the LMEM-based intervals have the most observations clustered near 0 (i.e., very little difference between the real $p$-value and the LMEM-based one), whereas within-subject and within-item intervals have longer positive tails (indicating more values that are substantially different from the real p-values). Figure 4 shows the correlations between the real p-values and the p-values suggested by LMEM-based intervals $(r=.994)$, within-subject intervals $(r=.973)$, and within-item intervals $(\mathrm{r}=.952)$.

A mixed effects model regressing the deviation (i.e., the amount from which the interval-based $p$-value differed from the real bootstrap $p$-value) on interval type ${ }^{4}$ found a marginal effect of interval type $\left(\chi^{2}(2)=4.69, p=.096\right)$, with mixed effects intervals marginally more accurate than by-subject Cousineau-Morey intervals $(b=0.03$, $S E=0.02, t=1.86)$ and significantly more accurate

\footnotetext{
${ }^{4}$ The model (in the syntax of the lme4 package) was as follows: diff IntervalType + ( IntervalType I Study/Experiment ) in which diff represents the difference between the interval-based p-value (i.e., the p-value based on what confidence level was needed to make intervals that overlapped by 58\%) and the real p-value based on bootstrapping the pairwise contrast. IntervalType represents whether mixed effect intervals, by-subject Cousineau-Morey intervals, or by-item Cousineau-Morey intervals were used. Study corresponds to a paper that one or more experiments were put together into, and Experiment corresponds to a single dataset within the study (most studies analyzed here consisted of one experiment, but some studies consisted of multiple experiments with the same design, or of an experiment including between-subject or between-item factors which I used to split the dataset into sub-experiments with fully crossed subjects and items). IntervalType was dummy coded with mixed-effect intervals as the baseline, and the main effect of IntervalType was tested via log-likelihood test comparing the above model with a model without that fixed effect (i.e., a model with only a fixed intercept and the random effects).
} 
Figure 3 - Smoothed kernel density of the differences between (a) the real p-values and the LMEM-interval-based pvalues (dotted blue line); (b) the real p-values and the within-subject-interval-based values (dashed red line); and (c) the real $\mathrm{p}$-values and the within-item-interval-based values (solid black line).

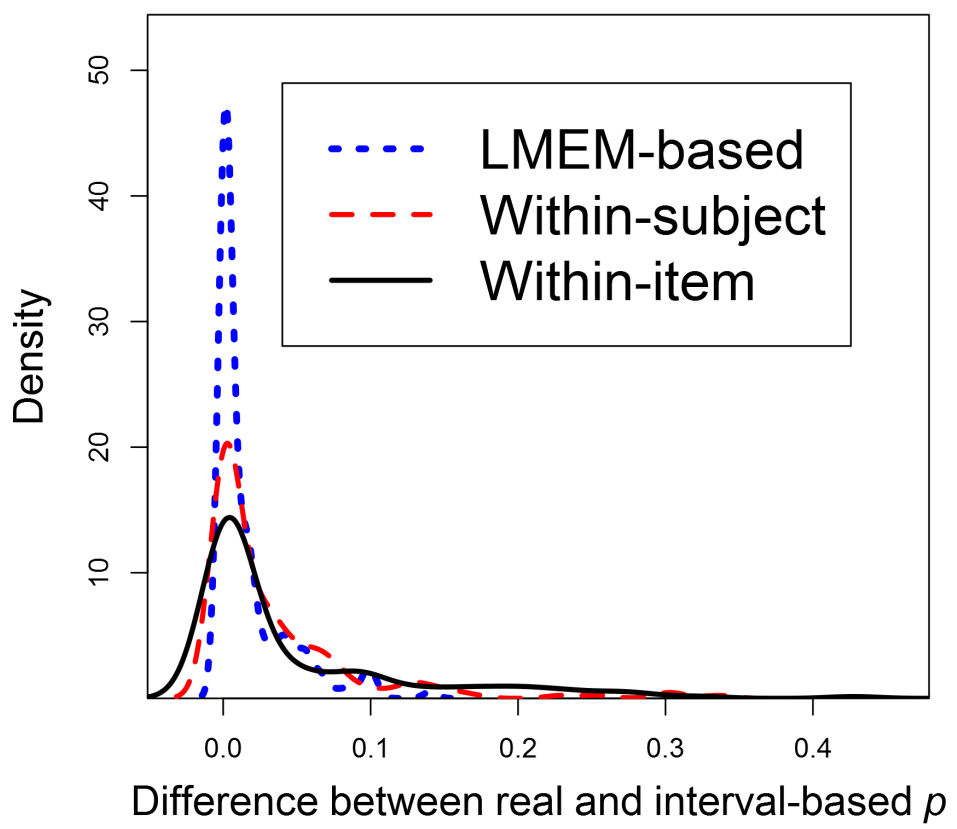

than by-item Cousineau-Morey intervals $(b=0.05, S E=$ $0.02, t=2.24$ ).

This advantage for mixed-effect intervals relative to within-subject and within-item intervals is also visible in the simulated data shown in Figure 2B. The 99\% withinsubject intervals (solid blue lines) in that figure suggest that the difference between the two conditions is not significant at the $\alpha=.01$ level, as they overlap by far more than $58 \%$ of the margin of error. The within-item intervals (solid black lines), on the other hand, suggest that the difference is significant at that level, as these intervals do not overlap at all. The mixed-effect model intervals, however, which are wider than the within-item intervals but narrower than the within-subject intervals, overlap by just about half the margin of error. This is consistent with the results of the direct statistical comparison, which gives a test statistic of $t=-2.17$ (see above), which corresponds to a $p$-value close to .01, which is consistent with the fact that it is the $99 \%$ mixed-effect intervals which overlap by close to $58 \%$ the margin of error. It should be noted, however, that this is a trend, rather than a guar- antee that LMEM-based intervals will align more closely with p-values; in fact, as can be seen in Supplementary File 4, there are some contrasts for which the p-values suggested by within-subject or within-item intervals align more closely with the real p-values, although these contrasts are in the minority.

\section{Conclusion}

The foregoing discussion presented a method for extending within-subject intervals to research designs with crossed random effects, and demonstrated that, in a sample of real datasets, the extent to which these intervals overlap (which is a common heuristic that practitioners use, often erroneously, to judge whether two means are different) corresponds more closely to the inferential statistics than previous within-subject and within-item types of intervals do. While these are not confidence intervals (i.e., they do not license conclusions about how often they will include a given parameter on repeated sampling), and they do not justify inferences about plausible parameter values (a limitation shared by bona fide confidence intervals), they 
Figure 4 - Scatterplots showing the correlation between real p-values and p-values suggested by each type of interval: LMEM-based intervals (top), within-subject intervals (middle), and within-item intervals (bottom).

LMEM - based intervals : $r=0.994$

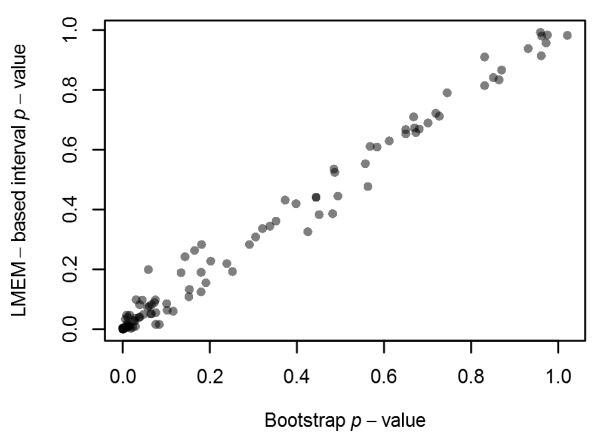

Within - subject intervals : $r=0.973$

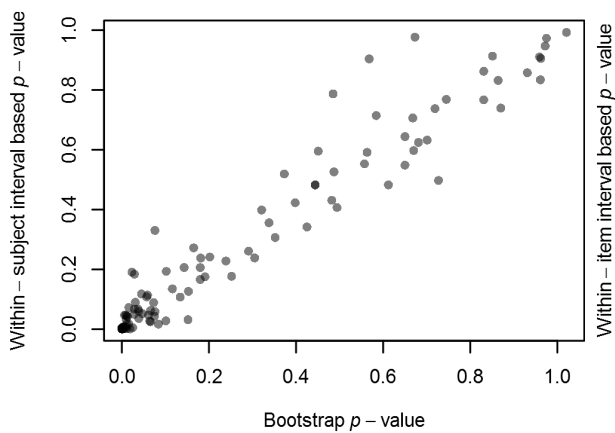

Within - item intervals : $r=0.952$

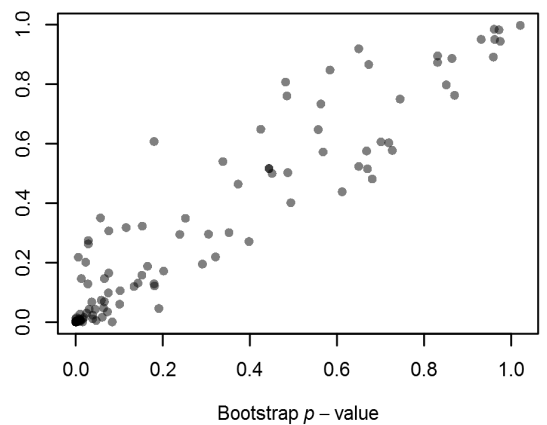

do offer a quick and roughly accurate means to visualize significant pairwise differences between a large number of conditions.

The present method is only discussed for relatively simple designs here. While it could be extended to multifactor designs and to designs including non-categorical (i.e., ordinal or continuous) predictors, these more challenging issues are beyond the scope of the present introduction. Furthermore, a unique challenge for this method is that random effects may also have different structures than those presented here. Random effects can be nested rather than crossed, or an experiment may have a combination of crossed and nested random effects. Likewise, some fixed effects may have corresponding random slopes and others (e.g., between-subject effects) may not. Here I have only considered designs that are fully within-subjects and within-items (when faced with datasets with betweensubject or between-item factors, I split them up into multiple datasets and calculated mixed effect intervals separately for each sub-dataset). The method could be used for designs that are not like this (for example, where a given subject contributes data to each condition, but a given item only appears in one condition), but the code given here would need to be adapted.

Because the method involves scaling, it is subject to the same concerns discussed by Franz and Loftus (2012), namely, the scaling may sometimes cause variance to propagate between conditions and may hide violations of the assumption of circularity. To my knowledge, every type of within-subject interval faces this problem; the only way around it is to plot true confidence intervals of differences (as noted by Franz and Loftus), rather than within-subject or LMEM-based intervals of individual conditions.

Visually evaluating whether two intervals overlap by
$58 \%$ of their average margin of error can sometimes be difficult. Practitioners who are interested could instead multiply the margins of error (i.e., half the interval) by $\sqrt{2}$. As described above in Footnote 5 (see also Pfister \& Jancyzk, 2013), this creates a difference-adjusted interval such that the difference between two means is likely to be significant at the given alpha level if one mean's interval does not contain the other mean. Just as for any other attempts to evaluate differences using two means' intervals, inferences made from this comparison are only rough estimates, and will be invalid if the two means have very different standard deviations or very different numbers of observations (Cumming \& Finch, 2005).

A practical concern is that calculating bootstrap confidence intervals takes a long time, particularly for models with many conditions. The datasets analyzed here took anywhere from several minutes to several hours to calculate percentile bootstrap confidence intervals. Other methods of bootstrapping may be faster (for instance, normal bootstrap confidence intervals can be estimated with far fewer replicates than percentile bootstrap confidence intervals), or intervals can be calculated using a confidence interval formula and estimating the degrees of freedom, but this method still is substantially slower than other within-subject interval methods. Some researchers may not want to spend this much extra time to obtain intervals that are only slightly more accurate, especially given that these intervals, just like other within-subject intervals, are still only a rough heuristic and will never be true confidence intervals. Nevertheless, for researchers who have sufficient time and want to present the most accurate heuristic data summary possible, while keeping in mind the limitations, this method may be a useful extension of other within-subject interval methods, as it does not re- 
quire aggregating across items or other random effects.

\section{Author's note}

I would like to thank Drs. Page Piccinini and Rory Turnbull for feedback on this work. Any errors are my own.

\section{References}

Baayen, H., Davidson, D., \& Bates, D. (2008). Mixed-effects modeling with crossed random effects for subjects and items. Journal of Memory and Language, 59, 390412. doi:10.1016/j.jml.2007.12.005

Baguley, T. (2012). Calculating and graphing within-subject confidence intervals for anova. Behavior Research Methods, 44, 158-175. doi:10.3758/s13428-011-0123-7

Barr, D., Levy, R., Scheepers, C., \& Tily, H. (2013). Random effects structure for confirmatory hypothesis testing: keep it maximal. Journal of Memory and Language, 68 , 255-278. doi:10.1016/j.jml.2012.11.001

Belia, S., Fidler, F., Williams, J., \& Cumming, G. (2005). Researchers misunderstand confidence intervals and standard error bars. Psychological Methocs, 10, 389396. doi:10.1037/1082-989X.10.4.389

Blouin, D. \& Rioppele, A. (2005). On confidence intervals for within-subjects designs. Psychological Methods, 10, 397-412. doi:10.1037/1082-989X.10.4.397

Chang, Y. \& Lane, D. (2016). Generalizing across stimuli as well as subjects: a non-mathematical tutorial on mixed-effects models. The Quantitative Methods for Psychology, 12, 201-219. doi:10.20982/tqmp.12.3.p201

Cousineau, D. (2005). Confidence intervals in withinsubject designs: a simpler solution to loftus and masson's method. Tutorials in Quantitative Methods for Psychology, 1, 42-45. doi:10.20982/tqmp.01.1.p042

Cumming, G. (2014). The new statistics: why and how. Psychological Science, 25, 7-29. doi:10 . 1177 / 0956797613504966

Cumming, G. \& Finch, S. (2005). Inference by eye: confidence intervals and how to read pictures of data. American Psychologist, 60, 170-180. doi:10.1037/0003066X.60.2.170

Davison, A. \& Hinkley, D. (1997). Bootstrap methods and their application. Cambridge: Cambridge University Press.
Franz, V. \& Loftus, G. (2012). Standard errors and confidence intervals in within-subjects designs: generalizing loftus and masson (1994) and avoiding the biases of alternative accounts. Psychonomic Bulletin \& Review, 19, 395-404. doi:10.3758/s13423-012-0230-1

Gould, L., McKibben, T., Ekstrand, C., Lorentz, E., \& Borowsky, R. (2016). The beat goes on: the effect of rhythm on reading aloud. Language, Cognition and Neuroscience, 31, 236-250.

Judd, C., Westfall, J., \& Kenny, D. (2012). Treating stimuli as a random factor in social psychology: a new and comprehensive solution to a pervasive but largely ignored problem. Journal of Personality and Social Psychology, 103, 54-69. doi:10.1037/a0028347

Loftus, G. \& Masson, M. (1994). Using confidence intervals in within-subject designs. Psychonomic Bulletin and Review, 1, 476-490. doi:10.3758/BF03210951

Morey, R. (2008). Confidence intervals from normalized data: a correction to cousineau (2005). Tutorials in Quantitative Methods for Psychology, 4, 61-64. doi:10. 20982/tqmp.04.2.p061

Morey, R., Hoekstra, R., Rouder, M., \& Wagenmakers, E. (2016). The fallacy of placing confidence in confidence intervals. Psychonomic Bulletin \& Review, 23, 103-123. doi:10.3758/s13423-015-0947-8

Pfister, R. \& Jancyzk, M. (2013). Confidence intervals for two sample means: calculation, interpretation, and a few simple rules. Advances in Cognitive Psychology, 9, 74-80. doi:10.5709/acp-0133-x

Rouder, J. \& Morey, R. (2005). Relational and arelational confidence intervals: a comment on fidler, thomason, cumming, finch, and leeman (2004). Psychological Science, 16, 77-79. doi:10.1111/j.0956-7976.2005.00783.x

Schaalje, G., McBride, J., \& Fellingham, G. (2002). Adequacy of approximations to distributions of test statistics in complex mixed linear models. Journal of Agricultural, Biological, and Environmental Statistics, 7, 512-524. doi:10.1198/108571102726

Tryon, W. (2001). Evaluating statistical difference, equivalence, and indeterminacy using inferential confidence intervals: an integrated alternative method of conducting null hypothesis statistical tests. Psychological Methods, 6, 371-386. doi:10.1037/1082-989X.6.4. 371

\section{Appendix A: Simulations to demonstrate the relationship between confidence interval overlap and p-values}

Some simple simulations can be used to test the validity of a given type of confidence interval. These in turn will be useful as diagnostics to examine whether LMEM-based intervals yield comparable results.

\section{The CI of paired differences}

Let's first consider the simplest case: a test of two paired samples (which is mathematically equivalent to a one-sample test against 0 , once the two vectors of paired samples are turned into one vector of differences). In this case a CI is both 
descriptive and inferential (to adopt Tryon, 2001, terms): it is the case both that $95 \%$ of $95 \%$ CIs will contain the true mean, and that a $95 \% \mathrm{CI}$ that does not cross zero indicates significance at $\alpha=.05$. Using simulations to illustrate the descriptive validity of the $\mathrm{CI}$ (i.e., that $95 \%$ of samples contain the true mean) is straightforward; since the within-subject CIs we discuss below are not true confidence intervals, however, I will only use simulations to illustrate the inferential validity of the CI (i.e., that the CI fails to include the null hypothesis value $\alpha \%$ of the time); for paired samples these are really just two different ways of stating the same problem. To verify the inferential validity of the CI, one simply needs to generate a large number of samples (of any sample size) from a given population where $\mu_{\text {difference }}=0$, and count the proportion of samples in which the CI fails to include 0 (i.e., the proportion of false positives). For a $95 \%$ CI this should be $5 \%$, and this is indeed what we observe with a large number of samples. Listing 2 was used to run this simulation.

Also note that in this case a CI and a $p$-value (from a $t$-test) are mathematically related, and one can be derived from the other. The $p$-value is simply the confidence level of the largest CI that just barely touches zero. This value can be straightforwardly calculated from the formula for the margin of error (i.e., half of the CI) where qt () is the quantile function (which finds the critical $t$ value for a given significance level and degrees of freedom; here the significance level is adjusted for a two-tailed test):

$$
\frac{S D_{x}}{\sqrt{n}} \cdot q t(1-\alpha / 2, n-1)=\bar{x}
$$

This can be algebraically solved for $\alpha$ (where pt() is the cumulative distribution function, the inverse of the quantile function: for a given t-value it returns a significance level):

$$
\alpha=2 \cdot\left(1-p t\left(\frac{\bar{x}}{S D_{x} / \sqrt{n}}, n-1\right)\right)
$$

Notice that this is, in fact, the $t$-test formula. Thus it is straightforwardly demonstrable that a $p$-value corresponds to the confidence level for the widest CI that just touches zero (or, specifically, $100 \times(1-p)$ corresponds to that confidence level). This can also be confirmed with any random sample, as seen with Listing 3.

\section{Two within-subject intervals, for two paired samples}

Now we consider a more complicated case: within-subject intervals for the means of the two paired samples, rather than one CI for the difference. As has been described previously (e.g., Cumming \& Finch, 2005, , among others), a 95\% CI of a condition mean is not directly informative about differences between that condition mean and other condition means: i.e., directly looking at whether that condition's CI overlaps with another does not provide an inference about whether the two conditions' means differ at $\alpha=.05$. Instead, as noted by Cumming and Finch (2005), as long as each group is $n>10$ and the two groups have similar variance, then the two means can be roughly inferred to differ at about $\alpha=.05$ if the overlap between the two confidence intervals is about $58 \%$ of the margin of error (MoE; i.e., half the width of the CI).

This $.58 \times M o E$ rule only directly applies to normal CIs in the case of comparisons between two independent groups. For comparisons between two conditions of within-subject (paired) data, a within-subject interval is needed (Loftus \& Masson, 1994). Here I will illustrate this with simulated Cousineau-Morey intervals. Just as we demonstrated inferential validity for a one-sample test (i.e., a paired differences test) above by counting the proportion of CIs that were simulated from a population with $\mu_{\text {difference }}=0$ but failed to cross 0 , we can conduct a similar demonstration for the two-condition case by drawing a large number of paired samples and counting the proportion of simulations in which the sample Cousineau-Morey CIs overlap by less than $58 \%$ of the average margin of error. Again, for a $95 \%$ interval and a population in which $\mu$ difference $=0$, this should be approximately $5 \%$ of samples, and this is indeed the case in the simulations run by Listing 4 .

Just as we did above, we can also draw a relationship between the Cousineau-Morey interval and the $p$-value by noting that the $p$-value is related to the confidence level of the interval. In this case, rather than corresponding to the confidence level of the widest interval that just touches zero, now the $p$-value corresponds to the confidence level of the widest pair of intervals that just barely overlaps by $58 \%$ of the average margin of error. (In the case with only two conditions, after scaling the data according to the Cousineau-Morey procedure then the margins of error for the two conditions are equal, and thus also equal to the average margin of error.) Once again, this can be algebraically calculated (where $x_{1}$ is the lower of the two condition means, and $\mathrm{MoE}$ is the margin of error for each condition):

$$
\frac{\left(\bar{x}_{1}+M o E\right)-\left(\bar{x}_{2}-M o E\right)}{M o E}=.58
$$


which can be solved for MoE:

$$
M o E=\frac{\bar{x}_{2}-\bar{x}_{1}}{1.42}
$$

And, in turn, solved for $\alpha$ after plugging in the MoE formula (with the correction factor, based on Morey, 2008):

$S D_{x_{\text {scaled }}} / \sqrt{n} \cdot q t(1-\alpha / 2, n-1) \cdot \sqrt{2}$ :

$$
\alpha=2 \cdot\left(1-p t\left(\frac{\bar{x}_{1}-\bar{x}_{2}}{-1.42} \cdot \frac{\sqrt{n}}{S D_{x_{\text {scaled }}} \cdot \sqrt{2}}\right)\right)
$$

Finally, as we did above for differences, here we can demonstrate for any simulated sample that this widest pair of Cousineau-Morey intervals that just barely reaches $58 \%$ overlap also has a confidence level approximately corresponding to $100 \times(1-p)$. Listing 5 was used to verify this.

(Notice that here, because the $.58 \times M o E$ rule only approximates $\alpha=.05$ significance level, rather than exactly equaling it, the confidence levels do not exactly match up with the $\mathrm{p}$ value calculated by the $t$-test; in fact, they are conservative, slightly overestimating the actual $p$ value.)

In summary, for Cousineau-Morey intervals of two paired means, the validity of the interval can be confirmed by using simulations to show that the false alarm rate (for inferences based on the proportion of overlap between two intervals) in a population with a zero effect is close to the nominal $\alpha$ level, and that the significance levels that can be inferred from the $.58 \times M o E$ rule closely approximate (although do not exactly equal) the $p$-value that would be yielded from directly testing the difference. We can then apply these same diagnostics to crossed mixed-effect intervals.

\section{Appendix B: An R convenience function for calculating LMEM-based intervals.}

This function is also available in the supplementary material on the journal's web site.

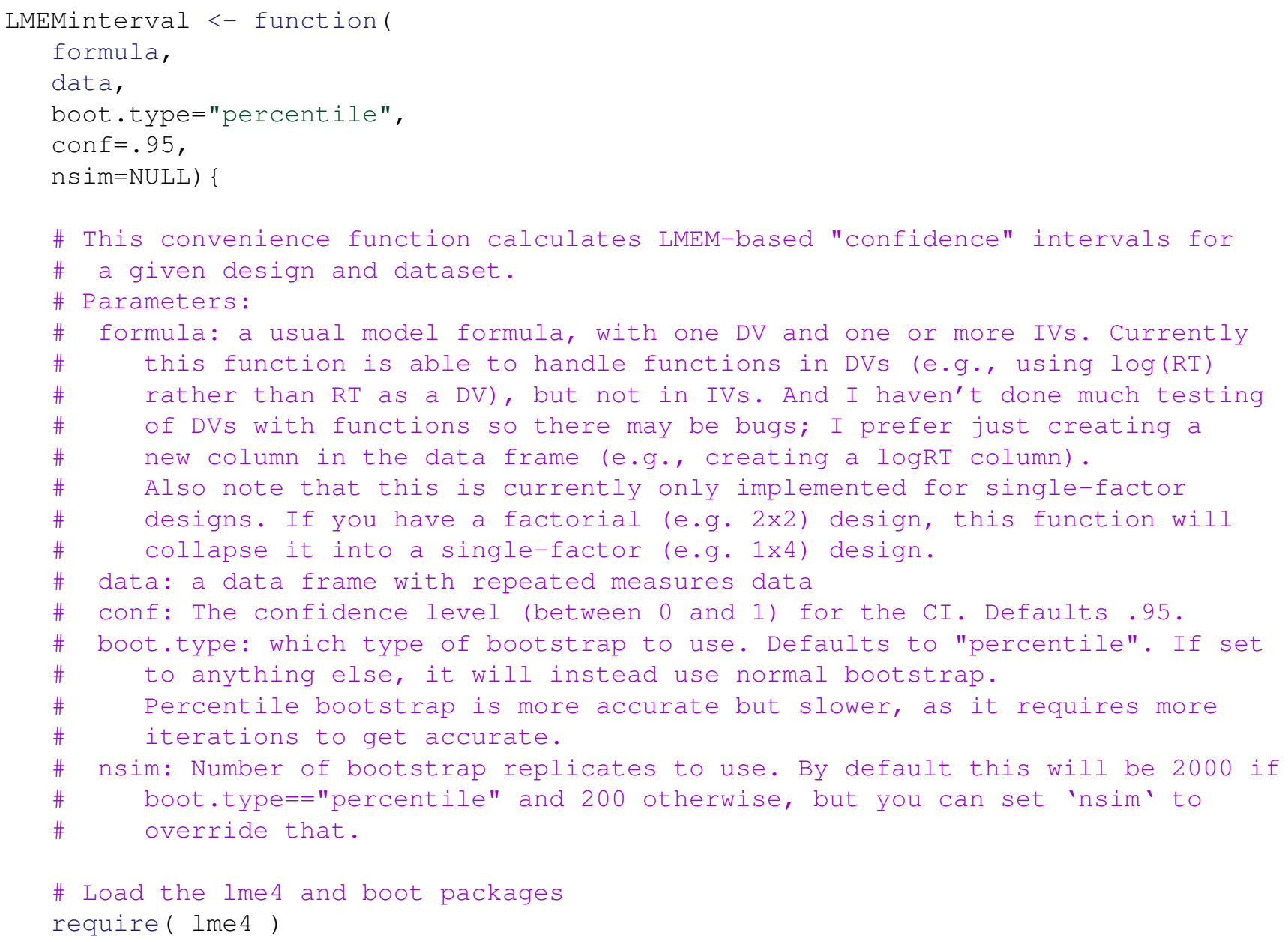




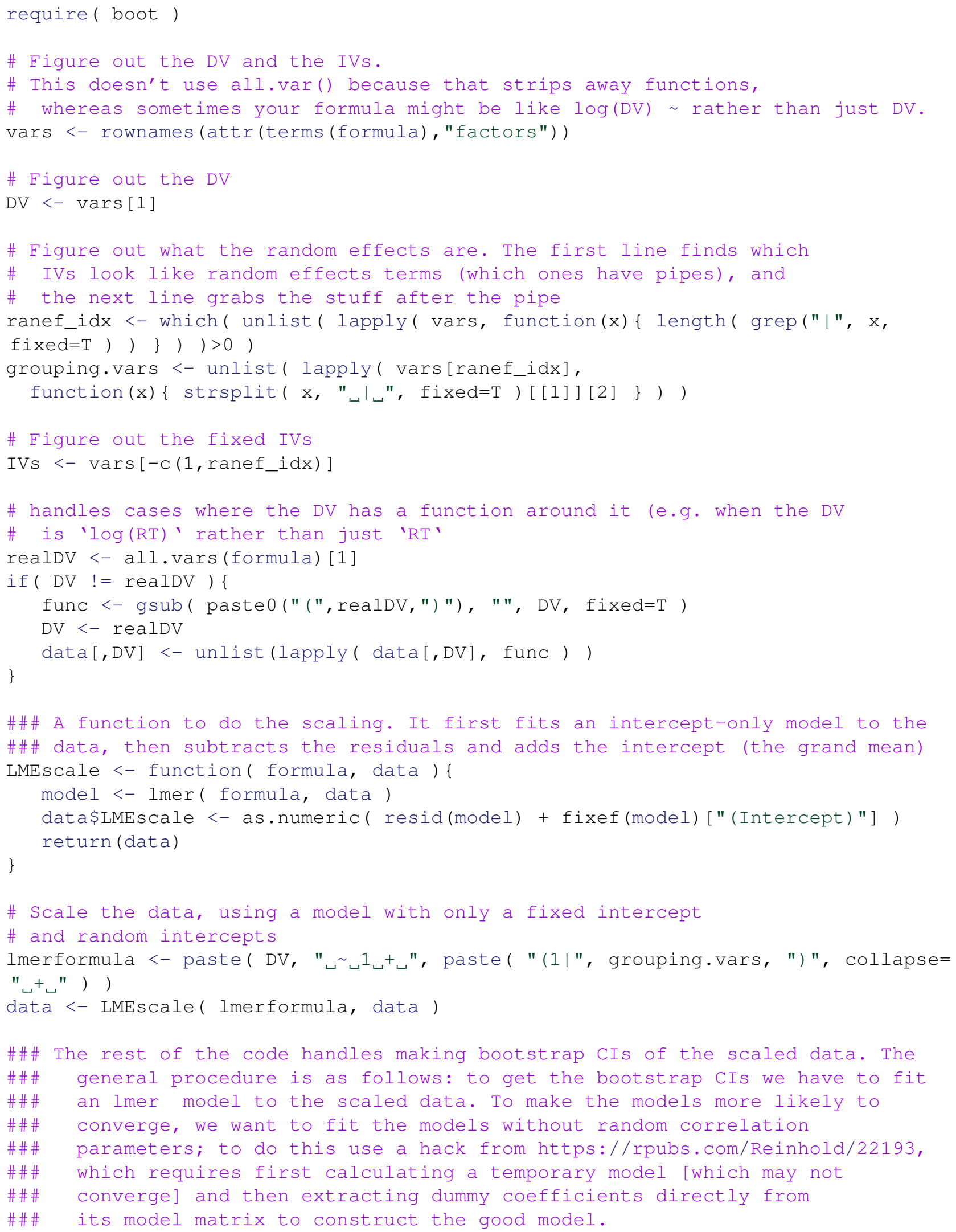




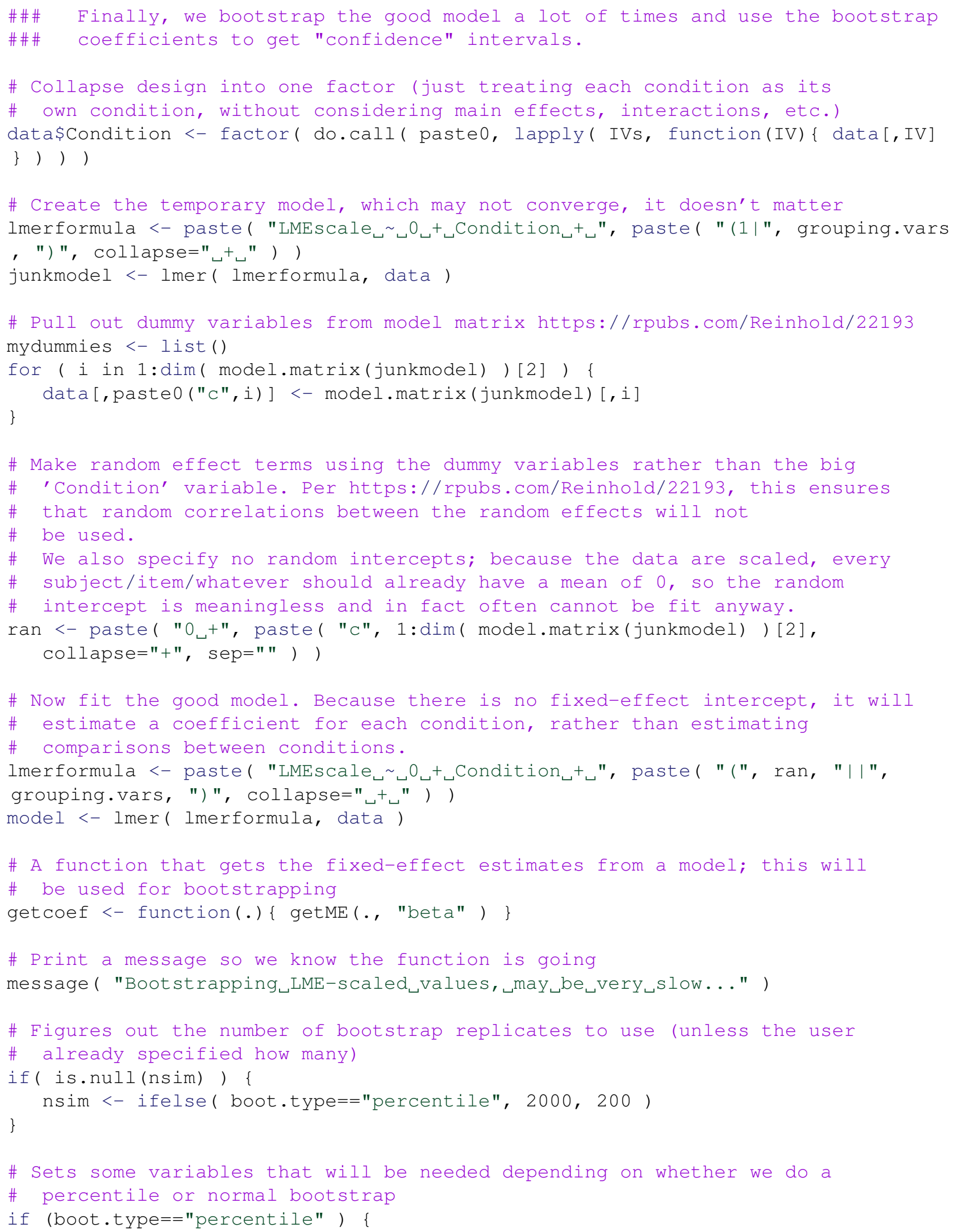




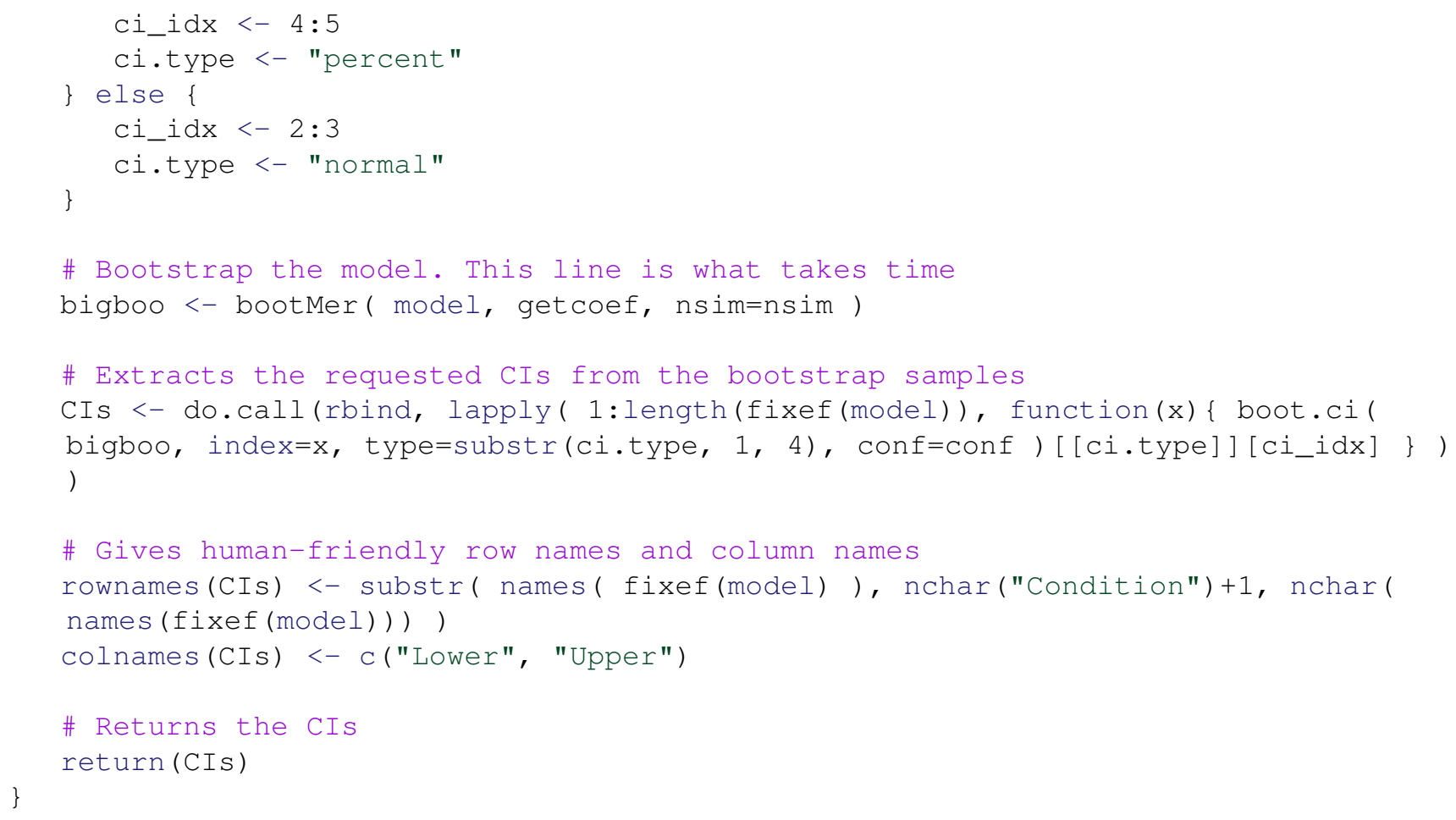

\section{Open practices}

- The Open Material badge was earned because supplementary material(s) are available on the journal's web site.

\section{Citation}

Politzer-Ahles, S. (2017). An extension of within-subject confidence intervals to models with crossed random effects. The Quantitative Methods for Psychology, 13(1), 75-94. doi:10.20982/tqmp.13.1.p075

Copyright ( 2017 , Politzer-Ahles. This is an open-access article distributed under the terms of the Creative Commons Attribution License (CC BY). The use, distribution or reproduction in other forums is permitted, provided the original author(s) or licensor are credited and that the original publication in this journal is cited, in accordance with accepted academic practice. No use, distribution or reproduction is permitted which does not comply with these terms.

Listings 1 to 5 follow. 
Listing 1 $\mathrm{R}$ code to demonstrate that scaled values based on the Cousineau approach are identical to residuals from ordinary least squares regression.

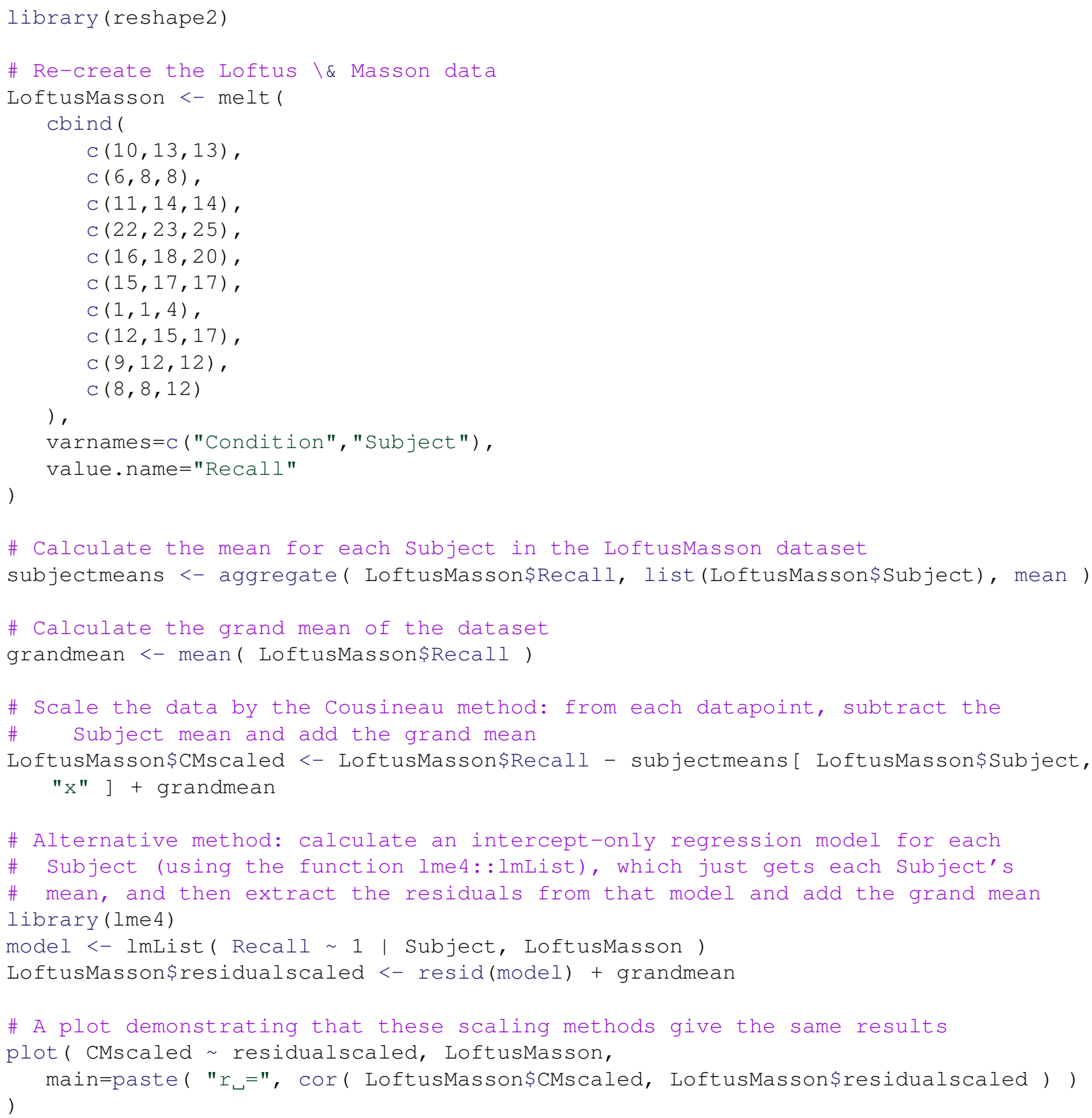


Listing 2 - R code to simulate a large number of samples and demonstrate that the proportion of samples in which the CI of the paired differences includes zero corresponds to the $p$-value.

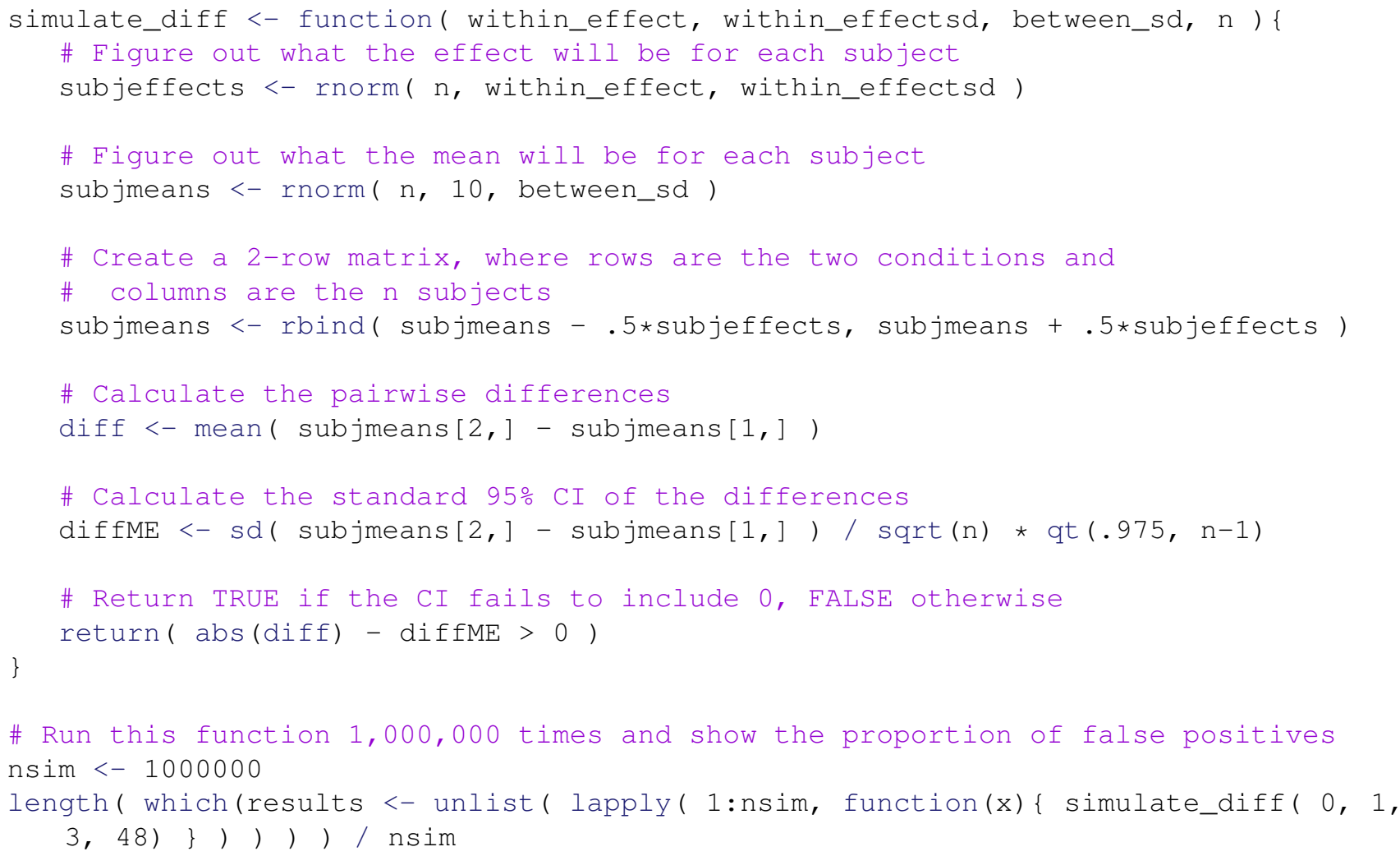

Listing 3 - R code to simulate a large number of samples and demonstrate the $p$-value is equal to the confidence level of the CI that just touches zero.

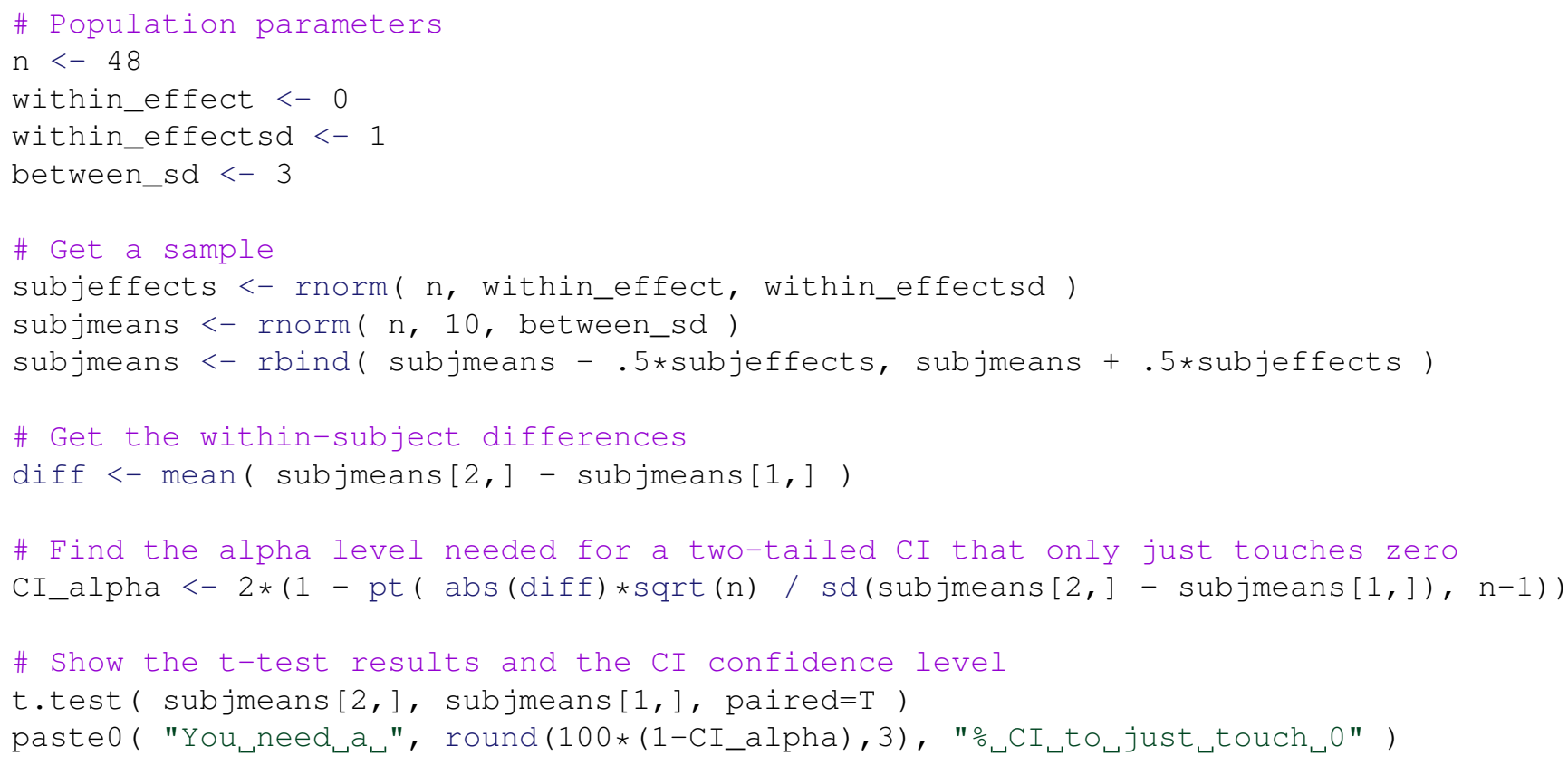


Listing 4 R code to simulate a large number of samples and demonstrate the $p$-value is close to the confidence level of the Cousineau-Morey intervals that overlap by just $58 \%$ of the average MoE.

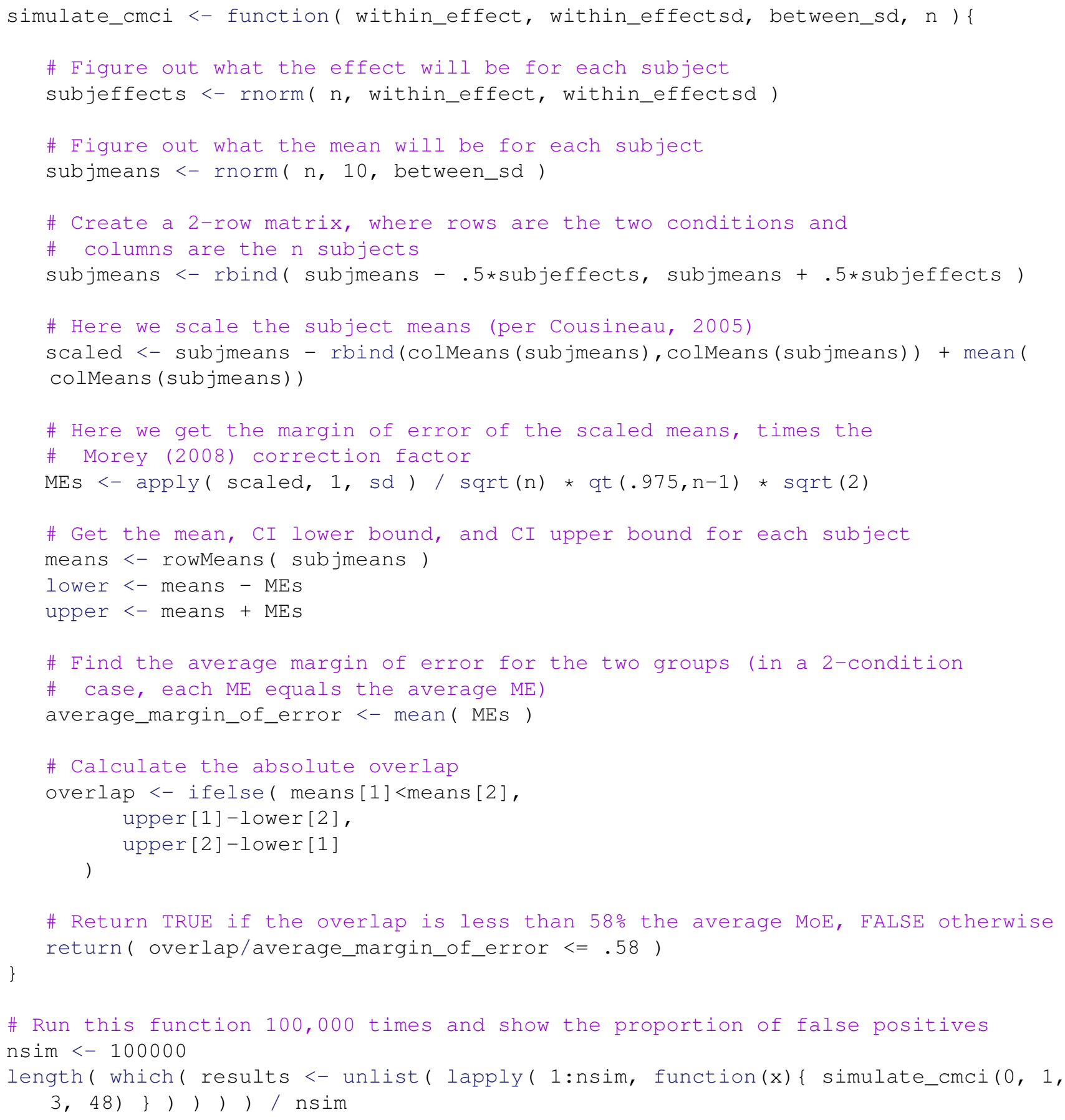


Listing 5 - R code to calculate a large number of Cousineau-Morey intervals at differnet confidence levels for a simulated dataset, and show that the confidence level of the intervals that overlap by just $58 \%$ of the average MoE closely corresponds to the $p$-value.

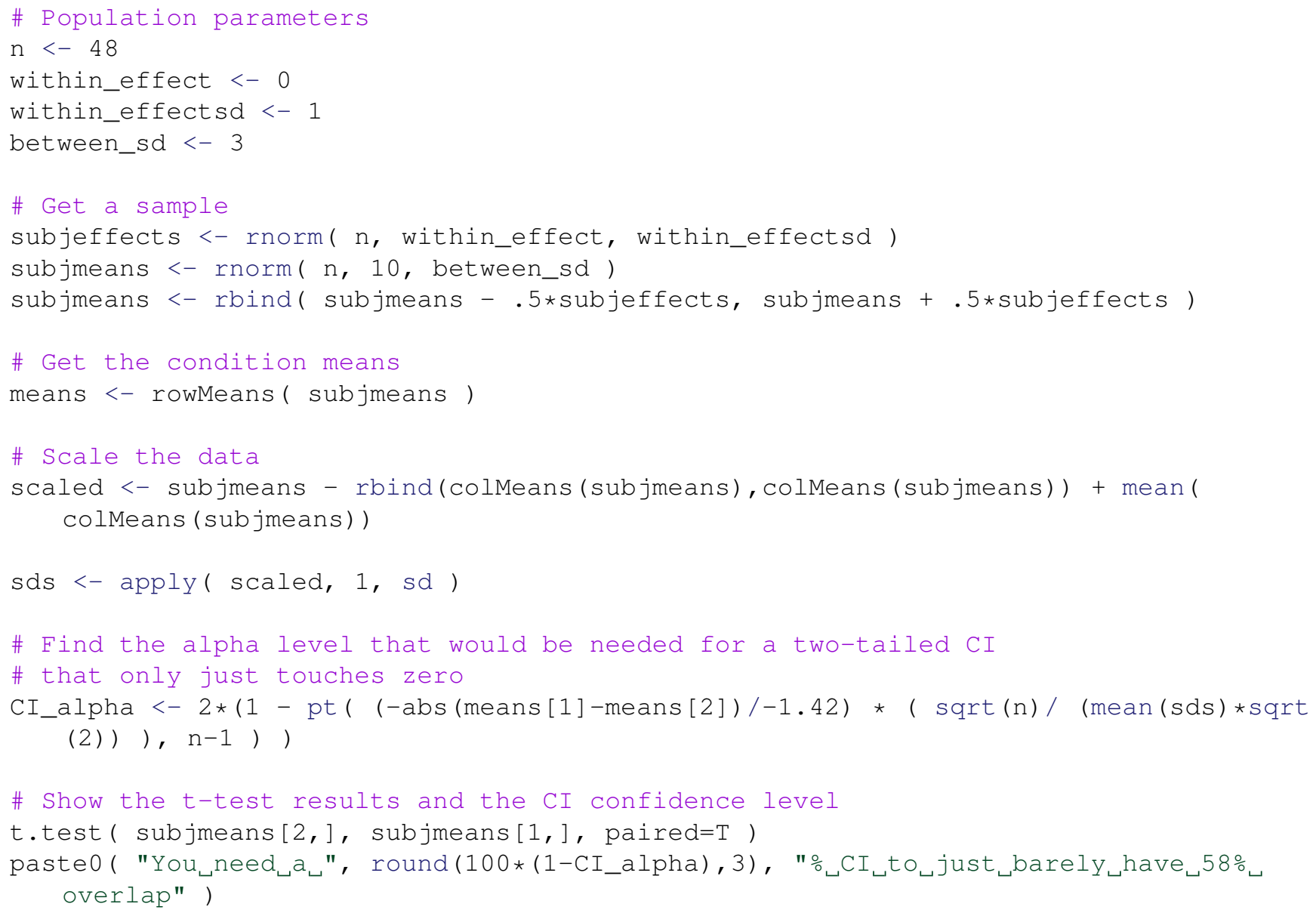

